\title{
Diseño de una lúdica en comunicación positiva como herramienta de capacitación en las organizaciones
}

\section{Design of a playful in positive communication as a tool for training in the organizations}

\author{
E. Quiroz, M. Cataño, J. T. Muñoz, L. Pulgarín y S. Pinzón
}

Recibido: mayo 15 de 2021 - Aceptado: junio 30 de 2021

\begin{abstract}
Resumen-La capacitación en comunicación positiva favorece la supervivencia de las organizaciones ante contextos cambiantes. Este proceso formativo requiere el uso de metodologías activas de aprendizaje, siendo la lúdica una alternativa. Este estudio tuvo como objetivo diseñar una lúdica en comunicación positiva como herramienta de capacitación en las organizaciones. Se utilizó la metodología propuesta por el Comité Estatal para la Ciencia y la Técnica de Rusia para el diseño de la lúdica, que fue validado a través del criterio de $\mathbf{1 0}$ expertos $(\mathrm{M}=40 \%-\mathrm{H}=60 \%)$, de los cuales el $80 \%$ tenían doctorado o maestría, en promedio 14 años de experiencia laboral y 12 en docencia e investigación. El $80 \%$ de los indicadores evaluados (claridad descriptiva, correspondencia objetiva, pertinencia y viabilidad) se consideran muy adecuados y el $20 \%$ (coherencia) bastante adecuado. Se concluye que la lúdica propuesta puede ser una herramienta útil para capacitar en
\end{abstract}

${ }^{1}$ Producto derivado de los proyectos de investigación: 1) Tecnoestrés y perspectiva de género: una lectura desde la salud mental, apoyado por la Universidad Católica de Pereira a través de la Convocatoria 874 de Minciencias 2020 "Convocatoria para el Fortalecimiento de Proyectos en Ejecución de CTeI en Ciencias de la Salud con Talento Joven e Impacto Regional" y 2) Incorporación de la Lúdica en los procesos de enseñanza y aprendizaje en la Ingeniería Industrial en tres Universidades del Eje Cafetero, financiado por la Universidad Católica de Pereira.

E. Quiroz, Universidad Católica de Pereira, Pereira, Colombia, email: eliana.quiroz@ucp.edu.co.

M Cataño, Universidad Católica de Pereira, Pereira, Colombia, email: mariano.catano@ucp.edu.co.

J.T Muñoz, Universidad Católica de Pereira, Pereira, Colombia, email: jennifer.munoz@ucp.edu.co.

L. Pulgarín, Universidad Católica de Pereira, Pereira, Colombia, email: laura2.pulgarin@ucp.edu.co.

S. Pinzón, Universidad Católica de Pereira, Pereira, Colombia, email: sebastian.pinzon@ucp.edu.co.

Cómo citar este artículo: Quiroz E., Cataño M., Muñoz J.T., Pulgarín L. y Pinzón, S. Diseño de una lúdica en comunicación positiva como herramienta de capacitación en las organizaciones, Entre Ciencia e Ingeniería, vol. 15 , no. 29 , pp. $46-53$, enero-junio 2021 .

DOI: https://doi.org/ 10.31908/19098367.2657.

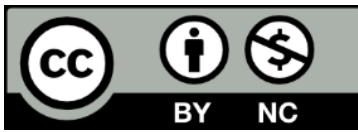

Attribution-NonCommercial 4.0 Intenational (CC By-NC 4.0) prácticas de comunicación positiva. Se recomienda su aplicación en organizaciones de distintos sectores.

Palabras clave - aprendizaje experiencial, bienestar laboral, comunicación corporativa, comunicación interna, juego serio.

Abstract - Training in positive communication favors the survival of organizations in changing contexts. This training process requires the use of active learning methodologies, with playful activities being an alternative. The objective of this study was to design a playful activity in positive communication as a training tool for organizations. The methodology proposed by the State Committee for Science and Technology of Russia was used for the design of the ludic, which was validated through the criteria of 10 experts $($ Female $=40 \%$ - Male $=60 \%)$, of which $80 \%$ had a doctorate or master's degree, on average 14 years of work experience and 12 years in teaching and research. The $80 \%$ of the indicators evaluated (descriptive clarity, objective correspondence, pertinence, and feasibility) are considered very adequate and $20 \%$ (coherence) quite adequate. It is concluded that the playful proposal can be a useful tool to train in positive communication practices. Its application may be recommended in organizations of different sectors.

Keywords - experiential learning, job well-being, corporate communication, internal communication, serious game.

\section{INTRODUCCIÓN}

$\mathrm{L}$ A formación del personal permite a las organizaciones enfrentar las transformaciones del mundo del trabajo, beneficiar su crecimiento económico y competitividad [1]. Así mismo, amplía la perspectiva del empleado al proporcionar elementos para ser agente en el logro de los objetivos organizacionales, por tanto, es un valioso recurso que contribuye a su realización personal y profesional [2]. De ahí que la formación sea, por excelencia, una de las prácticas de talento humano que mejores resultados individuales y organizacionales genera, debido a que no sólo permite ahorrar recursos a las empresas, sino que también aporta valor agregado a la salud laboral [3][4].

Promover el bienestar y la salud de los trabajadores debe ser un pilar estructural de la organización [5], dado que las 
personas son su activo más importante [6]. Lo anterior debe ser asumido desde una perspectiva estratégica, ya que las empresas que se preocupan por el bienestar de sus empleados, tienen mayores posibilidades de alcanzar óptimos resultados y sobrevivir ante contextos cambiantes [7].

Ahora bien, la formación en el ámbito empresarial es un proceso metódico que fortalece actitudes, conocimientos, competencias, destrezas y funciones que impactan el rendimiento laboral; se llevan a cabo mediante entrenamientos (inducciones), prácticas de desarrollo (crecimiento educativo o profesional que permite el ascenso entre cargos) y capacitaciones (enfocadas en habilidades para el desempeño de los puestos de trabajo).

Cabe señalar que la capacitación bajo métodos tradicionales es insuficiente, es imperioso acercar la pedagogía al ámbito organizacional a través del aprendizaje experiencial, que se caracteriza por ser dinámico, participativo e interactivo [8]. Para esto se recomienda elegir una metodología activa de aprendizaje, ésta se diferencia de las tradicionales porque requiere mayor participación y compromiso por parte del trabajador, quien asume un papel activo en la construcción de su propio conocimiento, lo que permite un aprendizaje significativo y duradero [9].

\section{A. Lúdica}

Dentro de las metodologías activas de aprendizaje se encuentra la lúdica, ésta presenta formas variadas para acceder a la información, por lo tanto, se deduce que va más allá del acto de jugar [10]; pese a que el término "lúdico" proviene de la palabra latina ludus, que significa juego o del término ludere, es decir, jugar [11], [12].

Posada González [13] resalta que la lúdica corresponde a una categoría macro que abarca el juego; adicional, la lúdica implica una sensación y actitud de gusto que acontece en relación con otros [14]. Esto ocurre porque el juego es un mecanismo natural arraigado al ser humano, que le permite desplegar su curiosidad, sentir placer y adquirir capacidades necesarias para desenvolverse en el mundo [15].

Por tanto, la lúdica consiste en el desarrollo de procesos de aprendizaje de forma interactiva y creativa, que facilitan la apropiación del conocimiento [14]. Incluso, es considerada como una representación de un determinado entorno real que da paso a la reflexión mediante el establecimiento de políticas, estrategias y toma de decisiones que se generan durante la interacción simulada [16]. De este modo, la lúdica trasciende las culturas e influye en el comportamiento, el aprendizaje y la comunicación de las personas [17].

La lúdica está centrada en el aprendizaje experiencial, por lo que facilita la adquisición de habilidades que difícilmente se obtienen bajo metodologías tradicionales [18], además favorece la satisfacción, motivación [8], habilidades cognitivas, motoras, sensoriales y sociales, que aumentan la calidad de vida, la salud mental, física y social [19], incrementa la creatividad, concentración, razonamiento [20], la participación, las habilidades en resolución de problemas [21], el clima, la eficiencia, la productividad laboral y la fidelización de empleados y clientes [22], [23].
Se debe agregar que las metodologías activas de aprendizaje son un apoyo para la innovación, el trabajo en equipo y la comunicación empresarial [20], [22], [23].

\section{B. Comunicación Positiva}

La comunicación es una herramienta eficaz para experimentar emociones positivas [24], dado que configura una evolución desde un plano intrapersonal a uno interpersonal [7], es decir, motiva las emociones en la relación consigo mismo y con otros, por ello, la comunicación gestiona el vínculo entre la existencia de las personas y la felicidad [25]. Esto apertura el término de Comunicación Positiva, la cual está soportada en los planteamientos de la psicología positiva [7] y es definida como aquel tipo de comunicación que, independientemente de los objetivos con los que se establezca, se enfoca en fomentar el bienestar de quienes participan en ella [26].

El papel de la comunicación positiva es brindar una mayor claridad en cuanto a la estructura organizacional y la asignación de responsabilidades [27], puesto que influye fuertemente en el comportamiento organizacional [28], inspira un ambiente de aprendizaje, incorpora a los trabajadores a los objetivos institucionales, amplifica las emociones positivas, los principios y valores que intervienen en la conexión con sus compañeros de trabajo [7], [29]. También enriquece la forma en que las personas entienden la estructura y el orden de su trabajo, lo que posibilita la obtención de resultados exitosos a nivel personal, grupal y organizacional [30]. Por esta misma línea, mejora el entendimiento que posee la organización del entorno y tiene un rol decisivo en el análisis interno frente al aprendizaje organizacional, de forma que su principal objetivo es forjar dinámicas positivas en la empresa [31].

Con este fin, la comunicación positiva no es un simple proceso de transmisión de información [29] o un conjunto de acciones de corte cosmético, realmente es un componente estratégico de la organización [32], una mediadora en las relaciones humanas, esto indica que, entre más positiva sea la comunicación de las personas, más positivas son sus relaciones [26]. De esta forma, la comunicación es una de las esferas que involucran al desarrollo humano y un eje principal en la calidad de vida [33].

En este estudio, la comunicación positiva se asume desde la perspectiva conceptual del modelo HERO (HEalthy \& Resilient Organizations), en español, modelo de organizaciones saludables y resilientes, dicho modelo plantea tres componentes interrelacionados: (I) los recursos y prácticas organizacionales saludables, que conducen a (II) trabajadores y equipos saludables, preparados para alcanzar el éxito empresarial al estar inmersos en una cultura y un ambiente laboral positivo, donde se generan (III) resultados organizacionales saludables, esto equivale a productos y servicios de excelente calidad [34]. En este orden de ideas, la gestión de la comunicación positiva se entiende como una práctica organizacional saludable. 


\section{Propuesta de una lúdica en comunicación positiva}

Para los líderes de Talento Humano, la gestión de la comunicación es una de las prácticas organizacionales saludables más útiles y de mayor uso [30], incluso en las pequeñas y medianas empresas [35]-[37]. Lo anterior sugiere que es importante que se implementen prácticas positivas, de hecho, reportes previos recomiendan diseñar prácticas y sumar evidencia que sean insumos para las intervenciones organizacionales que correspondan con las realidades propias de cada territorio [38], [5]. Por tanto, es necesario impulsar investigaciones que contribuyan a establecer y evaluar prácticas saludables (ejemplo: formación y capacitación en comunicación positiva), puesto que favorecen la consolidación de organizaciones saludables y resilientes [39].

Así, es una tarea inexcusable que las organizaciones den apertura a espacios de capacitación con sus trabajadores que los acerquen al desarrollo de habilidades para alcanzar una comunicación interna efectiva. Sin embargo, como ya se ha mencionado, no basta con emplear métodos tradicionales para alcanzar estos fines [8], se requiere de las metodologías activas de aprendizaje.

En este sentido, los estudios sobre diseños de lúdicas empresariales son limitados, al considerar que en Colombia el énfasis ha estado en lúdicas para estudiantes de Instituciones de Educación Superior [12], [16], [40], [41]; sin embargo, los desarrollos relacionados con la clase trabajadora muestran una evidencia insuficiente, especialmente en el fortalecimiento de la comunicación positiva. Por lo anterior, el objetivo de este estudio es diseñar una lúdica en comunicación positiva como herramienta de capacitación en las organizaciones.

\section{METODOLOGÍA}

\section{A. Diseño de la lúdica}

Los autores diseñaron una lúdica como herramienta didáctica para ser implementada en organizaciones de diferentes sectores. Esta busca desarrollar conceptos y reconocer la importancia de la comunicación positiva; así mismo, propiciar el uso de metodologías activas de aprendizaje en escenarios de capacitación en el contexto laboral. Lo anterior implica reconocer las particularidades de las diversas realidades de trabajo propias de cada entorno.

La lúdica consiste en recrear la cocina de un restaurante, los participantes deben gestionar, no solo los elementos físicos para la preparación de los alimentos, sino también los aspectos emocionales y comunicativos, los cuales pueden representar características de las relaciones interpersonales al interior de las organizaciones.

Para el desarrollo de la lúdica se requiere mínimo ocho participantes, quienes deben asumir diferentes roles como: administrador de restaurante, jefe de cocina, auxiliares de cocina, personas de oficios varios y un cliente. Para la ejecución de la lúdica se explica a los participantes sus funciones y el objetivo de la actividad, que consiste en entregar los pedidos completos y correctos dentro del tiempo de entrega establecido como promesa de valor al cliente.

Los productos del pedido pueden ser: pizza, burro o ensalada; cada preparación debe elaborarse en la cocina por los participantes. La transformación de los alimentos involucra tiempos establecidos de amasado, corte, horneado, limpieza y entrega.

Los materiales para el desarrollo de la lúdica son en su mayoría productos de papelería y manualidades que son reutilizables.

La lúdica consta de dos corridas, en ambos casos se elaboran los productos ya mencionados y se hace uso de los mismos recursos, sin embargo, en la segunda corrida se adicionan elementos que facilitan el control de las actividades y la mejora de los procesos en la cocina. Las operaciones inician con el pedido del cliente que se genera cada minuto; el producto a ordenar depende de una función de distribución de probabilidad a partir del lanzamiento de 2 dados.

El cliente verifica que los productos terminados estén completos, correctos y entregados a tiempo. Si una de las condiciones anteriores no se cumple, el pedido debe rechazarse. Al finalizar cada corrida, se determina si la organización cumplió con la promesa de valor.

Cada corrida tiene condiciones diferentes. En la primera, prevalece la comunicación verbal. El participante que asuma el rol de jefe de cocina debe simular rasgos propios de una persona agresiva sin que los demás participantes se enteren de esta indicación, por tanto, el jefe de cocina debe exigir la elaboración de cada plato mediante un tono fuerte y demandante. Además, sus expresiones corporales y verbales deben corresponder a una actitud con poca tolerancia hacia sus compañeros. Cabe destacar que el jefe de cocina debe hacer uso de su memoria para almacenar los pedidos realizados por el cliente, por ende, no va a contar con un recurso físico para escribir y así recordar el pedido.

Al finalizar la primera corrida se facilita un espacio de socialización y retroalimentación que permita de manera inductiva a través de los facilitadores, que los participantes lleguen a conclusiones importantes donde se involucren conceptos propios de la comunicación positiva.

En la segunda corrida, los participantes pueden utilizar herramientas adicionales que consideren importantes y necesarias para que la comunicación sea positiva. Adicionalmente, se pueden considerar ajustes en las instalaciones de la cocina con consentimiento de los facilitadores; la persona que asuma el papel de jefe de cocina debe simular rasgos propios de un líder transformacional, al comunicarse con su equipo propone la elaboración de cada plato mediante un tono cálido, comprensivo, empático y motivador. Además, sus expresiones corporales y verbales corresponden a una actitud tolerante, solidaria y asertiva, esta persona está dispuesta a ayudar con el desarrollo de las actividades de sus compañeros.

Durante las dos corridas participa un grupo de observadores, quienes tienen una lista de chequeo en comunicación positiva. Esta lista de chequeo contiene comportamientos observables que dan cuenta de la apropiación de la comunicación positiva en los participantes. Algunos comportamientos observables son: establece contacto visual con su interlocutor, utiliza un tono de voz cálido, 
escucha las instrucciones dadas y ofrece retroalimentación al equipo de trabajo. Los comportamientos son evaluados en una escala: nunca, a veces y siempre, también incluye una columna para observaciones.

Al finalizar la actividad, se contempla la socialización de recomendaciones basadas en la evidencia que permiten la mejora de la comunicación positiva en equipos de trabajo, así mismo, se permite analizar, evaluar y comparar la experiencia vivida por los participantes dadas las condiciones de cada corrida. En esta socialización se retoman los comportamientos observados y documentados en la lista de chequeo, también se establecen semejanzas y diferencias entre la práctica simulada en la cocina y el escenario real de trabajo. El énfasis que se propone no está en los productos elaborados, sino en las dinámicas relacionales y en el papel de la comunicación en el cumplimiento de los propósitos que como equipo de trabajo se establecen.

\section{B. Validación de la lúdica}

Con el objetivo de determinar que la lúdica sea comprensible y pertinente para ser usada en los procesos de capacitación empresarial relacionados con la comunicación positiva, se utilizó la validación a través del criterio de expertos, que consiste en someter a valoración un instrumento $\mathrm{u}$ objeto de estudio a profesionales con un alto grado de conocimiento en un tema con el fin de validar una propuesta específica [42]-[45].

En cuanto a la selección de los expertos, se identificaron profesionales que acrediten estudios de postgrado, experiencia laboral en industria, docencia e investigación, así como conocimiento en el uso de la lúdica y comunicación organizacional.

De acuerdo con la metodología establecida, se envió un documento detallado con el diseño de la lúdica, este contenía instrucciones, descripciones, recursos e información necesaria para su ejecución.

Se adaptó un instrumento de validación para el diseño de la lúdica en comunicación positiva, este se compartió en un Formulario de Google que tenía al final un espacio de observaciones. Se utilizó la metodología propuesta por el Comité Estatal para la Ciencia y la Técnica de Rusia, con el objetivo de determinar el coeficiente de competencia $(\mathrm{K})$ del experto. Para el cálculo de este coeficiente fue necesario indagar sobre el nivel de conocimiento de los expertos $(\mathrm{Kc})$ sobre el tema de interés y las fuentes que les permiten argumentar sus criterios (Ka) [16], [46].

Para definir el coeficiente de conocimiento $(\mathrm{Kc})$ se incluyó en la encuesta una pregunta de autoevaluación de cada experto con respecto a la experticia en temas lúdicos, utilizando una escala que mide el grado de conocimiento de 1 a 10 , los valores resultantes se multiplicaron por 0,1 [16], [47], [48].

Para conocer el grado de influencia desde el cual se sustenta la fundamentación y conocimiento de la lúdica $(\mathrm{Ka})$, se establecieron fuentes como: análisis teórico, experiencia obtenida, trabajos de autores nacionales, trabajos de autores internacionales, el propio conocimiento y la intuición, utilizando una escala tipo Likert: alto, medio y bajo [16].

Tanto para el coeficiente de argumentación (Ka) como para el coeficiente de competencia $(\mathrm{K})$ se empleó una escala de valoración por rangos: alta si los coeficientes se encuentran entre 0,8 y 1 , media si los coeficientes se encuentran entre 0,5 y 0,79 y baja si los coeficientes se encuentran por debajo de 0,5 .

Por último, se agregaron indicadores a la encuesta para valorar la lúdica (tabla I), se utilizó una escala Likert: muy adecuado, bastante adecuado, adecuado, poco adecuado y no adecuado [16].

TABLA I INDICADORES DE VALORACIÓN

\begin{tabular}{|c|l|}
\hline Indicador & \multicolumn{1}{c|}{ Explicación } \\
\hline $\begin{array}{c}\text { Claridad } \\
\text { descriptiva }\end{array}$ & $\begin{array}{l}\text { El diseño de la lúdica es comprensible a partir de los } \\
\text { conceptos que la integran y de las relaciones que se } \\
\text { establecen entre ellos. }\end{array}$ \\
\hline $\begin{array}{c}\text { Correspondencia } \\
\text { objetiva }\end{array}$ & $\begin{array}{l}\text { El diseño de la lúdica se relaciona claramente con el } \\
\text { dominio de la comunicación positiva. }\end{array}$ \\
\hline Pertinencia & $\begin{array}{l}\text { El diseño de lúdica propuesto da respuesta a la } \\
\text { necesidad de construcción de una herramienta didáctica } \\
\text { que sirva para aportar en el proceso de capacitación } \\
\text { empresarial relacionado con la comunicación positiva. }\end{array}$ \\
\hline Viabilidad & $\begin{array}{l}\text { El uso de la lúdica es posible en escenarios } \\
\text { organizacionales que requieran de capacitación en } \\
\text { comunicación positiva. }\end{array}$ \\
\hline Coherencia & $\begin{array}{l}\text { El diseño de la lúdica es lógico y consistente en cuanto } \\
\text { a la metodología propuesta para abordar el tema de } \\
\text { comunicación positiva. }\end{array}$ \\
\hline
\end{tabular}

\section{RESULTADOS}

Se identificaron 13 posibles expertos, de los cuales 11 aceptaron participar en el estudio.

Una vez efectuada la lectura del documento del diseño de la lúdica por parte de los expertos, fue aplicada la encuesta y con los resultados se procedió a determinar el coeficiente de competencia $(\mathrm{K})$ de cada uno de ellos como se muestra en la tabla II, éste se obtiene a partir de la ecuación (1).

$$
k=\frac{1}{2}\left(k_{c}+k_{a}\right)
$$

Uno de los expertos se descartó debido a que su coeficiente de competencia fue de 0,4 , correspondiente a una valoración baja: demuestra bajo dominio, conocimiento y experiencia en lúdicas; de incluirse un experto con valoración baja, afectaría el concepto que se emita respecto al diseño propuesto.

Entre las características de los expertos seleccionados se encontró que el $40 \%$ eran mujeres y el $60 \%$ hombres, el $80 \%$ tenían formación de doctorado o maestría. En promedio tenían 14 años de experiencia laboral y 12 años de experiencia docente e investigativa.

Posteriormente se realizó el procesamiento de la información suministrada por parte de los expertos con respecto a la valoración de la lúdica, para esto se tuvieron en cuenta los indicadores establecidos en la tabla I. Se realizó una tabla de frecuencias absolutas donde se relaciona el resultado obtenido para cada indicador y para cada categoría (tabla III). 
TABLA II

COEFICIENTE DE COMPETENCIA DE EXPERTOS

\begin{tabular}{|c|c|c|c|c|}
\hline Experto & Kc & Ka & K & Valoración \\
\hline $\mathbf{1}$ & $\mathbf{0 , 4}$ & $\mathbf{0 , 9}$ & $\mathbf{0 , 6 5}$ & Media \\
\hline $\mathbf{2}$ & $\mathbf{0 , 9}$ & $\mathbf{1}$ & $\mathbf{0 , 9 5}$ & Alta \\
\hline $\mathbf{3}$ & $\mathbf{0 , 8}$ & $\mathbf{0 , 8}$ & $\mathbf{0 , 8}$ & Alta \\
\hline $\mathbf{4}$ & $\mathbf{0 , 8}$ & $\mathbf{0 , 9}$ & $\mathbf{0 , 8 5}$ & Alta \\
\hline $\mathbf{5}$ & $\mathbf{0 , 7}$ & $\mathbf{0 , 9}$ & $\mathbf{0 , 8}$ & Alta \\
\hline $\mathbf{6}$ & $\mathbf{0 , 5}$ & $\mathbf{1}$ & $\mathbf{0 , 7 5}$ & Media \\
\hline $\mathbf{7}$ & $\mathbf{0 , 6}$ & $\mathbf{0 , 9}$ & $\mathbf{0 , 7 5}$ & Media \\
\hline $\mathbf{8}$ & $\mathbf{0 , 9}$ & $\mathbf{0 , 8}$ & $\mathbf{0 , 8 5}$ & Alta \\
\hline $\mathbf{9}$ & $\mathbf{0 , 6}$ & $\mathbf{0 , 7}$ & $\mathbf{0 , 6 5}$ & Media \\
\hline $\mathbf{1 0}$ & $\mathbf{0 , 8}$ & $\mathbf{0 , 9}$ & $\mathbf{0 , 8 5}$ & Alta \\
\hline
\end{tabular}

TABLA III

FRECUENCIAS ABSOLUTAS DEL RESULTADO DE LA EVALUACIÓN POR CRITERIO DE EXPERTOS

\begin{tabular}{|c|c|c|c|c|c|c|}
\hline Indicador & MA & BA & A & PA & NA & Total \\
\hline $\begin{array}{c}\text { Claridad } \\
\text { descriptiva }\end{array}$ & $\mathbf{6}$ & $\mathbf{4}$ & $\mathbf{0}$ & $\mathbf{0}$ & $\mathbf{0}$ & $\mathbf{1 0}$ \\
\hline $\begin{array}{c}\text { Correspondencia } \\
\text { objetiva }\end{array}$ & $\mathbf{6}$ & $\mathbf{3}$ & $\mathbf{1}$ & $\mathbf{0}$ & $\mathbf{0}$ & $\mathbf{1 0}$ \\
\hline Pertinencia & $\mathbf{5}$ & $\mathbf{4}$ & $\mathbf{1}$ & $\mathbf{0}$ & $\mathbf{0}$ & $\mathbf{1 0}$ \\
\hline Viabilidad & $\mathbf{5}$ & $\mathbf{5}$ & $\mathbf{0}$ & $\mathbf{0}$ & $\mathbf{0}$ & $\mathbf{1 0}$ \\
\hline Coherencia & $\mathbf{5}$ & $\mathbf{4}$ & $\mathbf{0}$ & $\mathbf{1}$ & $\mathbf{0}$ & $\mathbf{1 0}$ \\
\hline
\end{tabular}

Donde:

MA: Muy adecuado.

BA: Bastante adecuado.

A: Adecuado.

PA: Poco adecuado.

NA: No adecuado.

Con el fin de determinar los intervalos estadísticos de cada categoría, se halló la inversa de la curva normal para cada valor de la tabla de frecuencias absolutas, se calcularon las sumatorias de las filas para cada indicador y las sumatorias de las columnas para cada categoría. El promedio de la sumatoria de las columnas arrojó como resultado los puntos de corte para cada categoría. Después se calculó el valor de $\mathrm{N}$ como se muestra en la ecuación (2).

$$
N=\frac{\text { Sumatoria de sumas de filas por indicador }}{\text { (Número de categorías } * \text { Número de preguntas) }}=\frac{49,77382}{25}=1,9909528
$$

El siguiente paso consistió en realizar una resta entre el valor de $\mathrm{N}$ y el promedio de la sumatoria de las filas, lo que permitió obtener el valor de cada indicador, que a su vez es ubicado dentro del rango del intervalo acotado definido por los puntos de corte correspondientes.

A partir de los resultados obtenidos en la tabla IV, se evidencia que los valores de la columna $\mathrm{N}$-promedio se encuentran en los intervalos de las dos primeras categorías, esto indica que para los expertos la metodología lúdica fue muy adecuada en cuanto a claridad descriptiva, correspondencia objetiva, pertinencia y viabilidad; con relación a la coherencia fue bastante adecuada como herramienta de capacitación de la comunicación positiva en las organizaciones.

Adicionalmente, varios expertos expresaron que la lúdica está bien diseñada y estructurada, considerándola como un buen aporte al entorno de formación y capacitación empresarial. También, consideraron que es una herramienta útil para conocer cómo la comunicación positiva puede influir en la generación de ambientes laborales óptimos que conlleven a una mejor productividad.

Por otro lado, los expertos realizaron recomendaciones orientadas a enfocar de manera específica las preguntas de reflexión y cierre de la lúdica respecto a la importancia de la comunicación positiva. Consideraron relevante aclarar conceptos y términos a los participantes, entendiendo que la lúdica se puede aplicar en cualquier escenario empresarial y que no necesariamente tienen conocimientos en comunicación o administración.

TABLA IV

DETERMINACIÓN DE LOS PUNTOS DE CORTE

\begin{tabular}{|c|c|c|c|c|c|c|c|}
\hline Indicador & Muy adecuado & Bastante adecuado & Adecuado & Poco adecuado & Suma & Promedio & N-promedio \\
\hline Claridad descriptiva & 0,25335 & 4,01281 & 4,01281 & 4,01281 & 12,29178 & 3,07294 & $-1,08199$ \\
\hline Correspondencia objetiva & 0,25335 & 1,28155 & 4,01281 & 4,01281 & 9,56052 & 2,39013 & $-0,39918$ \\
\hline Pertinencia & 0,00000 & 1,28155 & 4,01281 & 4,01281 & 9,30717 & 2,32679 & $-0,33584$ \\
\hline Viabilidad & 0,00000 & 4,01281 & 4,01281 & 4,01281 & 12,03843 & 3,00961 & $-1,01866$ \\
\hline Coherencia & 0,00000 & 1,28155 & 1,28155 & 4,01281 & 6,57591 & 1,64398 & 0,34697 \\
\hline Suma & 0,50669 & 11,87028 & 17,33279 & 20,06405 & 49,77382 & & \\
\hline Puntos de corte & 0,10134 & 2,37406 & 3,46656 & 4,01281 & & & \\
\hline \multirow[b]{2}{*}{ Intervalos } & 0,10134 & 2,47539 & 5,84061 & 7,47937 & & & \\
\hline & $<0,10134$ & 0,10134 - 2,47539 & $\begin{array}{c}2,47539 \text { - } \\
5,84061 \\
\end{array}$ & $\begin{array}{c}\mathbf{5 , 8 4 0 6 1}- \\
\mathbf{7 , 4 7 9 3 7} \\
\end{array}$ & & & \\
\hline
\end{tabular}


Finalmente, se sugirió abordar aspectos motivacionales dentro de la lúdica, al considerar que existe una relación directa con la comunicación positiva. De gestarse una comunicación que permita a un empleado sentirse bien, dada la actitud del jefe, éste es capaz intrínsecamente de apropiarse de su labor y trabajar mejor.

\section{CONCLUSIONES}

Los expertos encontraron que la metodología lúdica fue muy adecuada en cuanto a claridad descriptiva, correspondencia objetiva, pertinencia y viabilidad, con relación a la coherencia, encontraron que fue bastante adecuada como herramienta de capacitación de la comunicación positiva en las organizaciones. En esta medida, se concluye que la lúdica en comunicación positiva es clara, objetiva, pertinente, viable y coherente para generar aprendizajes significativos a través de la capacitación.

Cabe destacar que los expertos hicieron énfasis en el papel de la formación en los resultados organizacionales y en cómo esta metodología facilita el logro de aprendizajes significativos y duraderos. Lo anterior es posible a través de la experiencia que generan las lúdicas en el momento en que sean implementadas. En este caso, la posible aplicación de la lúdica aquí propuesta, invita a los trabajadores a adoptar prácticas y comportamientos asociados a la comunicación positiva. Tales conductas se traducen principalmente en criterios observables que se pueden hacer evidentes en las relaciones establecidas por los participantes durante la futura implementación del ejercicio, por ejemplo, el apoyo ofrecido a sus otros compañeros o la actitud y expresión adoptada durante los procesos de diálogo al efectuar las demandas de su rol.

Además, el concepto de los expertos concuerda con el rol protagónico que tiene la comunicación positiva para lograr ambientes laborales idóneos, los cuales no solo fortalecen la productividad, sino que impactan el bienestar laboral.

En esta misma línea, los antecedentes evidencian que las prácticas saludables, específicamente, las prácticas de comunicación positiva generan mejores resultados organizacionales y potencian el bienestar integral, la satisfacción personal y laboral, la motivación, el desempeño e incluso la felicidad de los empleados [7], [25], [30], [35], [36], [38].

Finalmente, investigar variables positivas es cada día más relevante, dado que son mayores los estudios entorno a fenómenos psicosociales negativos como el estrés, el síndrome de burnout, el absentismo, la adicción al trabajo, entre otros temas bajo esta perspectiva, en comparación con el bienestar, la satisfacción, la felicidad o el disfrute [30], [49]. Incluso cuando el concepto de salud no se limita a la ausencia de enfermedad, sino que según la Organización Mundial de la Salud [50] es un estado de completo bienestar a nivel físico, mental y social, que como se ha mencionado, permite alcanzar resultados positivos, generar soluciones ante los problemas y brindar a los trabajadores herramientas para afrontar dificultades [51].

La presente investigación tiene como limitación que la efectividad de la metodología lúdica se evaluó a través de expertos y no se aplicó en entornos reales de trabajo. En este caso, la aplicación se imposibilitó por las recientes medidas para la contención de la pandemia por Covid-19 y las restricciones de movilidad ocasionadas por las manifestaciones de protesta social en Colombia. Se recomienda que futuras investigaciones puedan implementar la lúdica aquí propuesta y evaluar el impacto de esta práctica en la formación y el desempeño del talento humano.

\section{AGRADECIMIENTOS}

Los investigadores agradecen a los expertos que generosamente evaluaron y retroalimentaron la lúdica en comunicación positiva.

\section{REFERENCIAS}

[1] Organización Internacional del Trabajo (OIT), "Conocimientos teóricos y prácticos y empleabilidad," 2021. https://www.ilo.org/global/topics/skills-knowledge-andemployability/lang--es/index.htm (accessed May 02, 2021).

[2] A. Coronel, "Capacitación del capital humano como una inversión para desarrollo," Eureka, vol. 7, no. 2, pp. 71-76, 2010.

[3] M. Salanova, S. Llorens, P. Torrente, and H. Acosta, "Intervenciones positivas para potenciar organizaciones saludables y resilientes," in Consultoria Organizacional, 2013, pp. 135-166.

[4] C. Parra-Penagos and F. Rodríguez-Fonseca, "La capacitación y su efecto en la calidad dentro de las organizaciones," Rev. Investig. Desarro. E Innovación, vol. 6, no. 2, pp. 131-143, 2016.

[5] E. Quiroz-González, J. D. Loaiza, and C. Mejía, "Bienestar en el trabajo: implicaciones y desafíos para las organizaciones saludables," in Organizaciones saludables. Una contribución desde la psicología y la comunicación, Universida., Pereira, 2020, p. 201.

[6] Organización Internacional del Trabajo (OIT), "Promoción de la salud y el bienestar en el trabajo (Seguridad y salud en el trabajo)," 2012, 2012. http://www.ilo.org/safework/areasofwork/workplace-healthpromotion-and-well-being/lang--es/index.htm (accessed May 12, 2021).

[7] J. A. Muñiz Velázquez and A. Álvarez-Nobell, "Comunicación positiva: La comunicación organizacional al servicio de la felicidad," Rev. Comun. Vivat Acad., no. 124, pp. 90-109, 2013.

[8] C. A. Prósperi, G. J. Sabarots, and M. G. Villa, "Uso de la gamificación para el logro de una gestión empresarial integrado," Perspect. las Ciencias Económicas y Jurídicas, vol. 6, no. 2, pp. 8397, 2016, doi: 10.19137/perspectivas-2016-v6n2a05.

[9] A. Fernández March, "Metodologías activas para la formación de competencias," Educ. Siglo XXI, vol. 24, pp. 35-56, 2006.

[10] A. M. Zuluaga Monsalve, "El juego y la lectura lúdica como herramientas pedagógicas para el uso de tecnologías de información y comunicación que potencien la construcción del pensamiento," Rev. ANFORA, vol. 9, no. 16, pp. 63-74, 2000, [Online]. Available: https://doi.org/10.30854/anf.v9.n16.2000.282.

[11] C. Carreras, "Del Homo Ludens a la gamificación," Quad. Filos., vol. 4, no. 1, pp. 107-118, 2017, doi: 10.7203/qfia.4.1.9461.

[12] D. C. López and L. A. Mejía, "Aplicación de una lúdica en el salón de clase para enseñanza de la ingeniería industrial . Caso ingeniería de métodos," Entre Cienc. e Ing., vol. 8, no. 15, pp. 90-99, 2014.

[13] R. Posada González, "La lúdica como estrategia didáctica," La lúdica como estrategia didáctica. Universidad Nacional de Colombia, Bogotá, p. 89, 2014.

[14] A. M. Escobar Martínez, J. Sánchez Trujillo, and A. Díaz Gómez, "La capacitación lúdica como herramienta comunicativa en la gestión del conocimiento.” Pontificia Universidad Javeriana, Bogotá, p. 265, 2009.

[15] M. D. Guzmán Franco, J. I. Aguaded Gómez, and A. Chaves Montero, "Las redes sociales como plataforma de teleformación a través de la metodología lúdica y participativa," in Educación y Tecnología Propuestas desde la investigación y la innovación educativa, Ediciones., R. Roig-Vila, Ed. Barcelona, 2016, pp. 485-486.

[16] C. M. Zuluaga-Ramírez and M. del P. Gómez-Suta, "Metodología lúdica para la enseñanza de la programación dinámica determinista en un contexto universitario," Entramado, vol. 12, no. 1, pp. 236-249, 2016, doi: http://dx.doi.org/10.18041/entramado.2016v12n1.23124.

[17] A. Torres-Toukoumidis, J. P. Zagal, and M. A. Pérez - Rodríguez, "Influencia del juego en la comunicación interna y externa de las 
organizaciones," in Comunicación institucional y cambio social. Claves para la comprensión de los factores relacionales de la comunicación estratégica y el nuevo ecosistema comunicacional, Ediciones., L. M. Romero-Rodríguez and M.-C. Rosalba, Eds. Sevilla, 2016, pp. 165-186.

[18] A. Redondo Castán, J. A. Pascual Ruano, Á. M. Gento Municio, and V. S. J. Rodríguez, "Gamificación en Ingeniería de Organización: 'Cubioska' un juego de Role en Entorno Web,' Dir. y Organ., vol. 69, pp. 62-81, 2019.

[19] F. Á. Tobón Marulanda, N. Gaviria García, and J. F. Ramírez Villegas, "La lúdica como método psicopedagógico: Una experiencia para prevenir la farmacodependencia en jóvenes," Av. en Psicol. Latinoam., vol. 30, no. 1, pp. 81-92, 2012 .

[20] S. Estellés Miguel, G. Rius Sorolla, M. E. Palmer Gato, and J. M. Albarracin Guillem, "Gamificación en formación en empresas," Dir. y Organ., vol. 62, pp. 35-40, 2017.

[21] A. Azadegan, J. C. K. H. Riedel, and J. Baalsrud Hauge, "Serious games adoption in corporate training," Lect. Notes Comput. Sci. (including Subser. Lect. Notes Artif. Intell. Lect. Notes Bioinformatics), vol. 7528 LNCS, pp. 74-85, 2012, doi: 10.1007/978-3-642-33687-4_6.

[22] C. Gallego Gómez and C. De Pablos Heredero, "La gamificación y el enriquecimiento de las prácticas de innovación en la empresa: Un análisis de experiencias," Intang. Cap., vol. 9, no. 3, pp. 800-822, 2013, doi: 10.3926/ic.377.

[23] E. Morales Corral, "El proceso de gamification como revitalizador socio-empresarial. Una visión desde la sociología de las organizaciones," in XII Congreso Español de Sociología. Grandes transformaciones sociales y nuevos desafios para la sociología, 2016, pp. 1-18.

[24] L. Baez Colegio and A. Álvarez-Nobell, "Comunicación positiva contra la desigualdad en América Latina: Anita Garibaldi - TECHO," Rev. la Red Académica Iberoam. Comun., vol. 7, no. 1, pp. 1-17, 2016.

[25] J. A. Muñiz-Velázquez, "La comunicación eudaimónica: Confluencias entre la comunicación y la felicidad desde una perspectiva eudaimónica," Commun. Soc. Chang., vol. 3, no. 1, pp. 48-76, 2015, doi: $10.17583 /$ csc.2015.1775.

[26] J. A. Muñiz Velázquez, "Comunicación positiva: comunicar para ser y hacernos felices," in Inteligencia emocional y bienestar II, Primera Ed., J. L. Soler Nages, L. Aparicio Moreno, O. Díaz Chica, E. Escolano Pérez, and A. Rodríguez Martínez, Eds. Zaragoza: Ediciones Universidad San Jorge, 2016, pp. 95-111.

[27] A. Castro-Martínez and P. Díaz-Morilla, "Comunicación interna y gestión de bienestar y felicidad en la empresa española," Prof. la Inf., vol. 29, no. 3, pp. 1-13, 2020, doi: 10.3145/epi.2020.may. 24.

[28] P. Capriotti, "Comunicación Corporativa. Una estrategia de éxito a corto plazo," Rep. C\&D - Capacit. y Desarro., vol. 13, pp. 30-33, 1999.

[29] J. Franganillo, L. Sánchez, M. Á. García Asensio, and A. Marqués, "Aprendizaje emocional y de valores en la formación universitaria, aplicado al grado de Comunicación Audiovisual de la Universidad de Barcelona," Rev. Lat. Comun. Soc., vol. 79, pp. 151-173, 2021, doi: 10.4185/RLCS-2021-1493.

[30] M. Salanova, S. Llorens, and I. M. Martínez, "Comunicación positiva," in Organizaciones Saludables, Primera Ed., España: Editorial Aranzadi S.A.U, 2019, pp. 167-178.

[31] L. Browning, G. H. Morris, and K. F. Kee, "The Role of Communication in Positive Organizational Scholarship," in The Oxford Handbook of Positive Organizational Scholarship, Oxford Uni., G. M. Spreitzar and K. S. Cameron, Eds. Londres, 2012, pp. 124.

[32] H. Abadía, E. Castaño, and E. Quiroz-González, "Comunicación organizacional: gestión interna, proyección y auditoría," in Educación y cultura: movilidades y perspectivas, Pereira: Universidad Católica de Pereira, 2018, p. 105.

[33] C. A. Calvache Mora, "Perspectiva sistémica de la comunicación humana y sus desórdenes," Rev. Ciencias La Salud, vol. 13, no. 3, pp. 327-329, 2015, [Online]. Available: http://revistas.urosario.edu.co/index.php/revsalud/article/download/436 5/3154.

[34] M. Salanova, S. Llorens, E. Cifre, and I. Martínez, "We Need a Hero ! Toward a Validation of the Healthy and Resilient Organization (HERO) Model," Gr. Organ. Manag., vol. 37(6), pp. 785 -822, 2012, doi: 10.1177/1059601112470405.

[35] H. Acosta, M. Salanova, and S. Llorens, “¿Qué prácticas organizacionales saludables son más frecuentes en las empresas? Un análisis cualitativo," Fòrum Recer., vol. 16, pp. 811-825, 2011, [Online]. Available: http://horarioscentros.uned.es/archivos_publicos/qdocente_planes/6267 49/7investigacipnhero.pdf.

[36] H. Acosta, V. Cruz-Ortiz, M. Salanova, and S. Llorens, "Healthy organization: analysing its meaning based on the HERO Model," Rev. Psicol. Soc., vol. 30, no. 2, pp. 323-350, 2015, doi: http://dx.doi.org/10.1080/21711976.2015.1016751 Downloaded.

[37] C. I. Hernández-Vargas, S. Llorens-Gumbau, and A. M. RodríguezSánchez, "Healthy employees and service quality in the healthcare sector," An. Psicol., vol. 30, no. 1, pp. 247-258, 2014, doi: 10.6018/analesps.30.1.143631.

[38] M. Salanova, S. Llorens, and I. Martínez, "Aportaciones desde la psicología organizacional positiva para desarrollar organizaciones saludables y resilientes," Papeles del Psicólogo, vol. 37, no. 3, pp. 177-184, 2016.

[39] M. Salanova, "Intervenciones Psicológicas Positivas en Organizaciones Saludables," 2020, [Online]. Available: https://www.youtube.com/watch?v=iZrhyV7WmrI.

[40] H. J. Hernández-Reinoza, C. Villota-Ibarra, and J. A. Jimenez-Builes, "Metodología lúdica para la enseñanza de la ingeniería de requisitos basada en esquemas preconceptuales," Rev. EIA, vol. 18, no. 35, pp. 115, 2021, doi: 10.24050/reia.v18i35.1394.

[41] C. R. Ruiz, I. A. Castiblanco, J. P. Cruz, L. C. Pedraza, and D. Londoño, "Juegos de simulación en la enseñanza de la Ingeniería Industrial: caso de estudio en la Escuela Colombiana de Ingeniería Julio Garavito," Entre Cienc. e Ing., vol. 12, no. 23, pp. 48-57, 2018, doi: 10.31908/19098367.3702.

[42] L. G. Juárez-Hernández and S. Tobón, "Análisis de los elementos implícitos en la validación de contenido de un instrumento de investigación,” Rev. Espac., vol. 39, no. 53, 2018, [Online]. Available: http://www.revistaespacios.com/cited2017/cited2017-23.pdf.

[43] R. M. Cooke, "Validation in the classical model," in Elicitation. International Series in Operations Research \& Management Science, vol. 261, L. Dias, A. Morton, and J. Quigley, Eds. Washington DC: Springer International Publishing, 2018, pp. 37-59.

[44] B. Mikulskienè, V. Medvedev, T. Vedlūga, and O. Navickienè, "Behaviour Patterns in Expert Recognition by Means of Structured Expert Judgement in Price Estimation in Customized Furniture Manufacturing.," in Modeling and Simulation of Social-Behavioral Phenomena in Creative Societies, N. Agarwal, L. Sakalauskas, and G. W. Weber, Eds. Springer, Cham, 2019.

[45] T. Ouariachi, J. Gutiérrez-Pérez, and M. D. Olvera-Lobo, "Criterios de evaluación de juegos en línea sobre cambio climático. Aplicación del método Delphi para su identificación.," Rev. Mex. Investig. Educ., vol. 22, no. 73, pp. 445-474, 2017.

[46] N. Oñate, L. Ramos, and A. Díaz, "Utilización del Método Delphi en la pronosticación: una experiencia inicial," Econ. Planif., vol. 3, no. 4, pp. 9-48, 1988.

[47] M. Cruz Ramírez and M. C. Martínez Cepena, "Perfeccionamiento de un instrumento para la selección de expertos en las investigaciones educativas," Rev. Electrónica Investig. Educ., vol. 14, no. 2, pp. 167179, 2012.

[48] A. Lopez Padron, M. O. Isaac Spinola, and F. Mohar Hernandez, "Evaluación de la calidad de la Educación Superior Politécnica Angolana: un modelo teórico-funcional de autoevaluación institucional," Meta Avaliação, vol. 10, no. 30, pp. 661-691, 2018, [Online].

Available: https://revistas.cesgranrio.org.br/index.php/metaavaliacao/article/view/ $1765 /$ pdf.

[49] M. Salanova and W. Schaufeli, El engagement en el trabajo Cuando el trabajo se convierte en pasión. Madrid: Editorial Alianza S.A, 2009.

[50] Organización Mundial de la Salud (OMS), "Constitución de la Organización Mundial de la Salud," in Conferencia Sanitaria Internacional, Jun. 1946, p. 100, Accessed: May 28, 2021. [Online]. Available: https://www.who.int/es/about/who-we-are/frequently-askedquestions.

[51] P. M. Le Blanc and W. G. M. Oerlemans, "Amplificación en el trabajo: Construyendo una fuerza de trabajo sostenible a través de intervenciones individuales de psicología positiva," Papeles de Psicólogo, vol. 37, no. 3, pp. 185-191, 2016. 


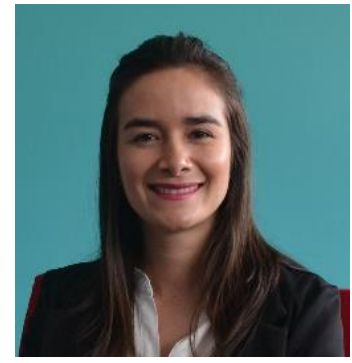

Eliana Quiroz González. Magíster en Gerencia de Talento Humano (2014, Universidad de Manizales, Manizales, Colombia), especialista en Gerencia del Talento Humano (2013, Universidad de Manizales, Manizales, Colombia), psicóloga (2011, Universidad Católica de Pereira, Pereira, Colombia). Docente e investigadora del programa de Psicología de la Universidad Católica de Pereira, integrante del grupo de investigación: Comunicación, Educación y Cultura. Áreas de interés investigativo: psicología organizacional positiva, capital psicológico y organizaciones saludables. ORCID: https://orcid.org/0000-0001-9061-8864
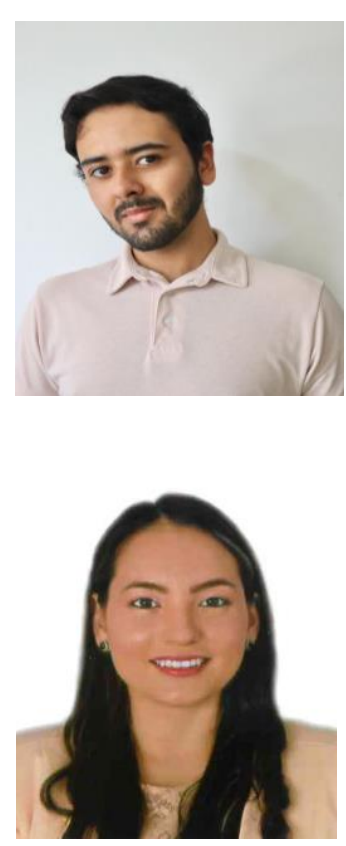

$\underline{8580}$
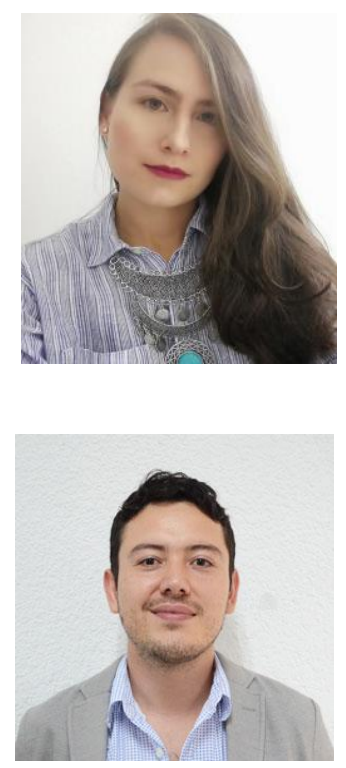
investigativo: Planeación de Ventas y Operaciones, Supply Chain Management, Gestión de Inventarios y Enseñanza en la Ingeniería. ORCID: https://orcid.org/0000-0003-1527-0399
Mariano Cataño Cárdenas. Estudiante de noveno semestre de Comunicación social Periodismo (Universidad Católica de Pereira), Diplomado en investigación e innovación (2020, Universidad Católica de Pereira). Joven investigador Minciencias 2020 - 2021, integrante del grupo de investigación: Comunicación, Educación y Cultura. Áreas de interés investigativo: comunicación organizacional, comunicación positiva, psicología positiva y organizaciones saludables. ORCID: https://orcid.org/0000$\underline{0002-2317-8261}$

Jennifer Tatiana Muñoz Jaramillo. Magíster en Pedagogía y Desarrollo Humano (2020, Universidad Católica de Pereira, Pereira, Colombia), psicóloga (2018, Universidad Católica de Pereira, Pereira, Colombia). Joven investigadora Minciencias 2020 - 2021, integrante del grupo de investigación: Comunicación, Educación y Cultura. Áreas de interés investigativo: psicología organizacional positiva, engagement $\mathrm{y}$ organizaciones saludables. ORCID: https://orcid.org/0000-0001-5245-

Laura Pulgarín Arias. Magíster en Administración Económica y Financiera (2020, Universidad Tecnológica de Pereira, Pereira, Colombia), ingeniera industrial (2013, Universidad Tecnológica de Pereira, Pereira, Colombia). Docente e investigadora de Ingeniería Industrial de la Universidad Católica de Pereira, integrante del grupo de investigación: Entre Ciencia e Ingeniería. Áreas de interés investigativo: enseñanza en la ingeniería, ORCID: https://orcid.org/00000002-4762-7498

Sebastián Pinzón Salazar. Magíster en Ingeniería (2021, Universidad EAFIT, Medellín, Colombia), especialista en Dirección de Operaciones y Logística (2017, Universidad EAFIT, Pereira, Colombia), ingeniero industrial (2013, Universidad Tecnológica de Pereira, Pereira, Colombia). Docente e investigador de Ingeniería Industrial de la Universidad Católica de Pereira, de la Universidad Católica de Pereira, Ciencia e Ingeniería. Áreas de interés 